\title{
A multiscale model of distributed fracture and permeability in solids in all-round compression
}

\author{
Maria Laura De Bellis ${ }^{\aleph}$, Gabriele Della Vecchia ${ }^{\dagger}$, Michael Ortiz ${ }^{\ddagger}$, Anna Pandolfi ${ }^{\dagger *}$ \\ ${ }^{\aleph}$ DII, Università del Salento, 73100 Lecce, Italy \\ $\uparrow$ DICA, Politecnico di Milano, 20133 Milano, Italy \\ ₹EAS, Caltech, Pasadena CA 91125, USA
}

\begin{abstract}
We present a microstructural model of permeability in fractured solids, where the fractures are described in terms of recursive families of parallel, equidistant cohesive faults. Faults originate upon the attainment of a tensile or shear resistance in the undamaged material. Secondary faults may form in a hierarchical organization, creating a complex network of connected fractures that modify the permeability of the solid. The undamaged solid may possess initial porosity and permeability. The particular geometry of the superposed micro-faults lends itself to an explicit analytical quantification of the porosity and permeability of the damaged material. The approach is particularly appealing as a means of modeling low permeability oil and gas reservoirs stimulated by hydraulic fracturing.
\end{abstract}

Keywords: Microstructured permeability; parallel faults; multi-scale permeability; analytical models.

\section{Introduction}

From the hydrogeological point of view, fractures and discontinuities are among the most important features of geological structures. In natural rock formations, fractures and other types of discontinuities facilitate storage and movement of fluids and represent the most ubiquitous and efficient conduits for fluid flows [1]. The availability of fault and fracture mappings in reservoirs is an important recent achievement in geology, but the understanding of the influence of these structures on fluid flows is still far from being satisfactory, in particular when the mechanical coupling is significant. The problem is compounded by the complexity of the topology and geometry of faults. Each group or class of faults is characterized by orientation, spacing, distribution, and connectivity, in manners that affect the entrapment of fluids, limiting or advantaging migration and flow of fluids in a given environment [2].

Clearly, the complexity of natural fracture networks is related to the stress state history, which is often unknown. Furthermore, cracks and fracture can evolve due to the action of gravity, superposed localized pressures, and shear tractions resulting from the viscosity of the flowing fluids. Fracture processes are often exploited in engineering technology, e. g., to improve and optimize hydraulic fractures for well stimulation in low permeability reservoirs, to prevent water or gas outburst into underground mines, to predict the integrity of reservoirs for underground $\mathrm{CO}_{2}$ sequestration or hazardous waste storage, and in other areas of application [3]. The excavation of underground structures in rock masses induces cracking accompanied, in general, by significant changes in flow and permeability due to the deterioration of geotechnical and hydrogeological properties [4].

Damage induced by mechanical or hydraulic perturbations influences the permeability of the rock mass, with significant effects on the pore pressure distribution. Modifications in the pore pressure, in turn, affects

\footnotetext{
${ }^{*}$ Corresponding author

Email address: anna.pandolfi@polimi.it (Anna Pandolfi ${ }^{\dagger}$ )

Preprint Submitted
} 
the mechanical response of the material by poromechanical coupling. According to experimental observations at the microscopic scale, fracture evolution in rocks can be interpreted essentially as a progressive damage accumulation process, characterized by nucleation, growth and coalescence of numerous cracks following changes in the external load or in the internal pore pressure [5, 6]. In the particular case of hydraulic fracturing, a stimulation technique used in petroleum industry to increase the oil/gas production in low permeability reservoirs, fractures are produced by the artificial increase of the fluid pressure in a borehole. From the theoretical point of view, it has been observed that the success of hydraulic fracturing is related to: i) the creation of a dense system of hydraulic cracks with limited spacing; and ii) the prevention or mitigation of localization instabilities [7].

The observation that intact rocks contain distributed flaws and cracks, arranged between particles of various shape, has motivated the use of fracture mechanics to study their organization and the conditions that promote their growth [8]. The description of two-dimensional flow through geometrically simplified fractures embedded in infinite porous media can be found in numerous studies, see, e. g., [9, 10]. In particular, the effective permeability of cracked materials has been analyzed theoretically, numerically, and empirically [11]. It is well known that standard approaches of fracture mechanics show limiting drawbacks related to the explicit treatment of cracks. For example, models that describe the damage of a material with the formation of isolated microcracks, that do not form a connected network, are not able to relate the evolution of damage to the enhancement of the permeability in a direct mechanistic fashion. This drawback has been tackled in recent studies [12, 13, 14], where intersection and interaction between curve-shaped fractures have been taken into account in numerical simulation of steady flow of fluids in anisotropic porous media.

As an alternative to fracture mechanics, continuum damage mechanics considers the averaged effect of microstructural changes, following a phenomenological approach able to reproduce hydro-mechanical responses during the progressive degeneration of rocks. A typical approach treats rock masses containing a large number of discontinuities as homogeneous, anisotropic porous media [15]. The cracks in the medium are assumed to follow a probability distribution function $\operatorname{PDF}(N, L, \Delta)$ in terms of crack orientation $N$, size $L$ and opening $\Delta$. A symmetric crack tensor associated to the permeability tensor of the cracked porous medium is derived though an averaging procedure. The principal directions of the permeability tensor are coaxial to those of the crack tensor. Thus, the first invariant of the crack tensor is proportional to the mean permeability, while the deviatoric part of the crack tensor is related to the anisotropic permeability.

In the framework of continuum mechanics, various methods have been developed to account for different factors in the theoretical evaluation of rock permeability, e. g., the coupled effect of flow, stress and deformation, the propagation of existing fractures, and the nucleation of new fractures. A simplified coupled hydro-mechanical continuum approach, based on the Biot's theory of fluid saturated porous media and on brittle-elastic solid with residual strength, was considered in a finite element model including damage combined with elastic unloading/reloading [16]. However, hydraulic anisotropy and internal state variables were not considered, thus the resulting permeability was treated as a scalar directly dependent on the stress state. With a similar approach, a damage model accounting for orientation, size and number of penny-shaped microcracks was used to predict the evolution of a scalar permeability in the context of the excavation of underground openings [17, 18]. However, as already noted, isolated microcracks fail to form a connected network and, consequently, it remains unclear how they can possibly contribute to the permeability of the medium.

A micromechanical point of view has been taken in [19] to assess the influence of local damage on the macroscopic hydro-mechanical response of porous media. The damage variables are related to the degradation of elastic properties and to the characteristics of the fracture network. Thus, the model is capable of describing the evolution of the porous network with deformation and the influence of deformation on permeability [20]. The model distinguishes between the natural pore network, sensitive to the deformation of the representative volume, and the crack network, enucleated in the damaged material, and assumes laminar fluid flow. The natural pore network is characterized by a Pore Size Distribution (PSD) curve, updated with the state variables and with the evolution of the cracks, and linked to the permeability. In spite of the anisotropic nature of the crack pattern, a scalar value of hydraulic conductivity is defined by integration of the PSD.

Coupling between deformation and fluid flow has been accounted for in a variety of ways. By consider- 
ing the volumetric strain as an additional controlling parameter, in [21] numerical simulations of permeability reduction (increase) upon elastic contraction (dilation) in rocks are presented. Permeability is considered as a scalar variable, although the approach accounts for flow in both matrix and fractures. The dependence of rock permeability on material deformation has been alternatively enforced in terms of crack opening. An elaborate coupled semi-empirical hydro-mechanical constitutive model accounting for anisotropic damage induced by cracks and modification in permeability in brittle rocks under deviatoric compressive stresses was proposed in [22]. The rock is regarded as a porous medium with embedded microcracks. Upon homogenization, the cracked material is treated as an equivalent porous medium where the permeability tensor is decomposed additively into initial and crack induced permeability tensors. Here again, how permeability can be induced despite the lack of connectedness of isolated microcracks remain essentially unexplained. Since the microcrack distribution is orientation dependent, the crack permeability tensor has to be anisotropic in nature and it is regarded as a function of crack number, orientation, radius, and average opening. The mechanical model is formulated in terms of linear elasticity and the crack propagation conditions are based on linear elastic fracture mechanics, without the support of a thermodynamical framework. The effects of damage on anisotropic permeability were discussed in [23] by adopting a relationship between macroscopic and microscopic aspects of damage, and exploiting micro-level analyses of flow through randomly generated crack networks.

Models of distributed damage and permeability based on abstract damage mechanics are, of necessity, empirical in nature and the precise meaning and geometry of the damage variables often remains undefined. In addition, the evolution of the damage variables and their relation to the deformation, stress and permeability of the rock mass is described by means of empirical laws that represent, at best, enlightened data fits. However, the permeability enhancement due to extensive fracturing of a rock mass depends sensitively on precise details of the topology, which needs to be connected, and geometry of the crack set, including the orientation and spacing of the cracks. In addition, the coupled hydro-mechanical response of the rock, especially when complex loading conditions and histories are of concern, is much too complex to yield to empirical data fitting.

Based on these considerations, in this paper we endeavor to develop a model of distributed fracturing of rock masses, and the attendant permeability enhancement thereof, based on an explicit micromechanical construction of connected patterns of cracks, or faults. The approach extends the multi-scale brittle damage material model introduced in [24], which was limited to mechanical damage. In contrast to abstract damage mechanics, the fracture patterns that form the basis of the theory are explicit and the rock mass undergoes throughout compatible deformations and remains in static equilibrium down to the micromechanical level. The fracture patterns are not arbitrary: they are shown in [24] to be optimal as regards their ability to relieve stress, and the inception, orientation and spacing of the fractures derive rigorously from energetic considerations. Following inception, fractures can deform by frictional sliding or undergo opening. The extension of the theory presented in this paper additionally accounts for fluid pressure by recourse to Terzaghi's effective stress principle. When the fluid pressure is sufficiently high, existing fractures can open, thereby contributing to the permeability of the rock mass. The attendant permeability enhancement can then be estimated using standard lubrication theory [25, 26, 27], resulting in a fully-coupled hydro-mechanical model.

The paper is organized as follows. We begin in Section 2 with illustrating the hydromechanical framework, recalling the basic equations and the Terzaghi's effective stress principle. In Section 3 we recall the main features of the dry material model developed in [24], introducing a pressure dependent behavior at fault inception. In Section 4 we derive analytically the permeability associated to the presence of faults in the brittle damage material model. In Section 5 we validate the material model by means of comparison with experimental results taken from the literature.

\section{Hydro-mechanical framework}

Deterioration of mechanical and hydraulic properties of rock masses and subsequent problems are closely related to changes in the stress state, formation of new cracks, and increase of permeability in porous media saturated with freely moving fluids. In fully saturated rocks, fluid and solid phases are fully interconnected 
and the interaction between fluid and rock is characterized by coupled diffusion-deformation mechanisms that convey an apparent time-dependent character to the mechanical properties of the rock.

The two governing equations of the coupled problem are the linear momentum balance and the continuity equation (mass conservation). The kinematic quantities that characterize this picture are the porous solid displacement $\boldsymbol{u}$ and the rate of fluid volume per unit area $q$. Hydro-mechanical coupling arises from the influence of the mechanical variables (stress, strain and displacement) on the continuity equation, where the primary variable is the fluid pressure, and from the influence of the hydraulic variables (pore pressure and seepage velocity) on the equilibrium equations, where the primary variables are the displacements.

\subsection{Fluid flow in porous media}

Fluid flow in porous media is governed by permeability, a property that measures the ability for fluids (gas or liquid) to flow through a porous solid material. The flow of a homogeneous fluid in a porous medium is characterized by its velocity $\boldsymbol{v}$, of magnitude $v$. The energy of a flowing fluid is traditionally measured in terms of total hydraulic head $h$, that for slow flowing fluids reads

$$
h=\frac{p}{\rho g}+z,
$$

where $\rho$ the fluid density, and $g$ the gravitational acceleration. The pressure head $p / \rho g$ is the equivalent gauge pressure of a column of water at the base of a piezometer. The elevation head $z$ expresses the relative potential energy.

Fluid flow across packed porous media is generally characterized by laminar regime (Reynolds number $\operatorname{Re} \leq 1)$ and by a drop of the hydraulic head $\boldsymbol{\nabla} h$ in the direction of the flow. Analytical models of fluid flow in rocks use constitutive relations that link the average velocity of the fluid across the medium to the hydraulic head drop. As representative example of constitutive relation, Darcy's law states that the discharge rate in a porous media is proportional to the hydraulic head gradient and inversely proportional to the fluid viscosity

$$
\boldsymbol{q}=-\boldsymbol{k} \frac{\rho g}{\mu} \nabla h,
$$

where $\boldsymbol{q}$ is the discharge rate, $\mu$ the fluid viscosity, and $\boldsymbol{k}$ the medium permeability tensor. Permeability is intrinsically related to the void topology and does not account for the properties of the fluid. In anisotropic media, permeability is a symmetric (consequence of the Onsager reciprocal relations) and positive definite (a fluid cannot flow against the pressure drop) second order tensor $\boldsymbol{k}$. Real eigenvalues of the permeability tensor are the principal permeabilities, and the corresponding eigenvectors indicate the principal directions of flow, i.e., the directions where flow is parallel to the pressure drop. Clearly, fractures modify the permeability tensor, introducing new preferential directions for fluid flow. Although affected by many factors, permeability is primarily related to the rock porosity (or void fraction) $n$, expressing the ratio between the volume of the voids $V_{V}$ and the total volume $V$ that accounts also for the solid volume $V_{S}$

$$
n=\frac{V_{V}}{V}=\frac{V_{V}}{V_{S}+V_{V}} .
$$

The rate of fluid volume $\boldsymbol{q}$ is linked to the porosity through the continuity equation, which for partially saturated voids reads

$$
\frac{\partial\left(n S_{r} \rho_{f}\right)}{\partial t}=-\nabla \cdot \rho_{f} \boldsymbol{q},
$$

where $\rho_{f}$ is the density of the fluid phase, $S_{r}$ the degree of saturation (i. e., the fraction of the fluid volume), $\nabla$ the divergence operator, and $\partial / \partial t$ the partial derivative with respect to time. Under the assumption of fully saturated voids and incompressible fluid, the continuity equation becomes

$$
\frac{\partial n}{\partial t}=-\nabla \cdot \boldsymbol{q} .
$$


We remark that the solid phase incompressibility assumption adopted in the present model is not affecting substantially the hydraulic behavior, mostly because the porosity of the matrix plays a minor role in the hydraulic conductivity of the material. In fact the porosity, and thus the permeability, is mostly imputable to the formation of faults, reducing the relevance of the matrix porosity.

\subsection{Mechanics equations}

In the absence of any occluded porosity, the solid grains forming the matrix generally undergo negligible volume changes. In keeping with standard assumptions in geomechanics, we consider the solid phase of the matrix incompressible, thus we regard the change of the volume of the matrix as a change of the volume of the voids of the matrix. This assumption is consistent with the adoption of the Terzaghi's theory, in lieu of the more sophisticated Biot theory, unnecessary for the foreseen applications of the model. Moreover, we consider fully saturated media.

The equations of mechanics in the finite kinematics framework are reported in Table 1 together with the corresponding equations in linearized kinematics. In the equations we denote with $\boldsymbol{\sigma}(\boldsymbol{P})$ the Cauchy (first Piola-Kirchhoff) stress, $\boldsymbol{b}(\boldsymbol{B})$ the spatial (material) body force vector, $\boldsymbol{t}(\boldsymbol{T})$ the spatial (material) surface tractions, $\boldsymbol{n}(\boldsymbol{N})$ the spatial (material) outward normal to the boundary, $\boldsymbol{\sigma}^{\prime}\left(\boldsymbol{P}^{\prime}\right)$ the effective stress, $p$ the pore pressure, $\boldsymbol{\varepsilon}=\operatorname{sym} \boldsymbol{\nabla} \boldsymbol{u}$ the small strain tensor, and $J$ the jacobian of the deformation gradient $\boldsymbol{F}$

$$
\boldsymbol{F}=\frac{\partial \boldsymbol{x}}{\partial \boldsymbol{X}}
$$

where $\boldsymbol{x}$ and $\boldsymbol{X}$ are the spatial and material coordinates, respectively.

Table 1: Mechanics and hydraulic equations

\begin{tabular}{ccc}
\hline Equation & Linearized kinematics & Finite kinematics \\
\hline \hline Linear momentum balance & $\operatorname{div} \boldsymbol{\sigma}+\boldsymbol{b}=\underline{0}$ & $\operatorname{Div} \boldsymbol{P}+\boldsymbol{B}=\underline{0}$ \\
Boundary conditions & $\boldsymbol{\sigma} \boldsymbol{n}=\boldsymbol{t}$ & $\boldsymbol{P} \boldsymbol{N}=\boldsymbol{T}$ \\
Terzaghi's principle & $\boldsymbol{\sigma}=\boldsymbol{\sigma}^{\prime}+p \boldsymbol{I}$ & $\boldsymbol{P}=\boldsymbol{P}^{\prime}+p J \boldsymbol{F}^{-T}$ \\
Solid constitutive law & $\boldsymbol{\sigma}^{\prime}=\boldsymbol{\sigma}^{\prime}(\boldsymbol{\varepsilon})$ & $\boldsymbol{P}^{\prime}=\boldsymbol{P}^{\prime}(b \boldsymbol{F})$ \\
Darcy's law & $\boldsymbol{q}=-\boldsymbol{k} \rho g \nabla h / \mu$ & $\boldsymbol{q}=-\boldsymbol{k} \rho g \nabla h / \mu$ \\
Porosity & $n=n_{0}+\Delta \varepsilon_{v}$ & $n=1-\left(1-n_{0}\right) / J$ \\
\hline
\end{tabular}

In finite kinematics, the Eulerian porosity defined in Eq. (2) is naturally associated to the jacobian of the deformation gradient $J=\operatorname{det} \boldsymbol{F}=V / V_{0}$,

$$
n=\frac{1}{J} \frac{V_{v}}{V_{0}}=1-\frac{1}{J}\left(1-n_{0}\right),
$$

see Appendix A for details of the derivation. Note that for very low values of $n_{0}$ Eq. (3) may provide negative values of porosity for $J<1$, thus a zero lower-bound must be enforced in calculations. We remark that the solid phase incompressibility assumption adopted in the present model is not affecting substantially the hydraulic behavior of the medium, since the porosity of the matrix plays a minor role in the hydraulic conduction of the model. In fact the porosity change, and thus the permeability change, is mostly due to the formation of faults, reducing the relevance of the porosity of the matrix. 


\section{Dry brittle damage model}

The brittle damage model presented in [24] is characterized by a homogeneous matrix where nested microstructures of different length scale are embedded. At each level (or rank) $k$ of the nested architecture, microstructures assume the form of families of cohesive faults, characterized by an orientation $\boldsymbol{N}_{k}$ and a uniform spacing $L_{k}$, see Fig. 1(a) In keeping with well established mathematical procedures used to treat free discontinuity problems, the constitutive model for the brittle damage is derived with a thermodynamically consistent approach, by assuming the existence of a free energy density which accounts for reversible and dissipative behaviors of the material.

\subsection{Kinematics}

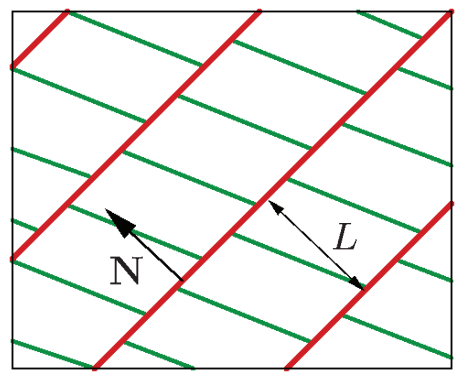

(a) Reference configuration

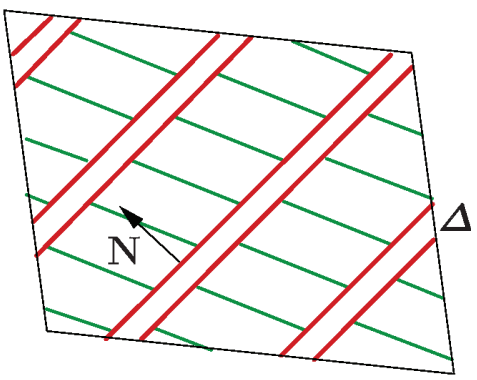

(b) Current configuration

Figure 1: Inelastic kinematics of the fault system. The opening displacement $\Delta$ applied to all the faults at distance $L$ leads to a deformed configuration characterized by the inelastic deformation gradient $\boldsymbol{F}^{\mathrm{f}}$.

The key of the brittle damage model is given by the kinematic assumptions. We begin by considering the particular case of a single family of fault planes of normal $N$ and spacing $L$, and later extend the behavior to recursive nested families. The total deformation gradient $\boldsymbol{F}$ of the material is assumed to decompose multiplicatively into a part $\boldsymbol{F}^{\mathrm{m}}$ pertaining the uniform deformation of the matrix, and a second part $\boldsymbol{F}^{\mathrm{f}}$ describing the discontinuous kinematics of the cohesive faults, i. e.,

$$
\boldsymbol{F}=\boldsymbol{F}^{\mathrm{m}} \boldsymbol{F}^{\mathrm{f}} .
$$

The deformation gradient $\boldsymbol{F}^{\mathrm{f}}$ can be easily linked to the kinematic activity of the faults. Consider a material vector $d \boldsymbol{X}$, shorter than the system size but longer than the internal scale $L$, that spans two material points $A$ and $B$ in the material configuration Fig $1(\mathrm{a})$. The number $m$ of faults traversed by the vector is

$$
m=\frac{1}{L} d \boldsymbol{X} \cdot \boldsymbol{N}
$$

Let us now apply an opening displacement $\Delta$ to each fault, Fig 1(b) In the spatial configuration the two points $A$ and $B$ are joined by the vector $d \boldsymbol{x}$ given by

$$
d \boldsymbol{x}=d \boldsymbol{X}+m \boldsymbol{\Delta}=d \boldsymbol{X}+\frac{1}{L}(d \boldsymbol{X} \cdot \boldsymbol{N}) \boldsymbol{\Delta}=\left(\boldsymbol{I}+\frac{1}{L} \boldsymbol{\Delta} \otimes \boldsymbol{N}\right) d \boldsymbol{X},
$$

where we set

$$
\boldsymbol{F}^{\mathrm{f}} \equiv \boldsymbol{I}+\frac{1}{L} \Delta \otimes N
$$

Once $\boldsymbol{N}$ and $L$ are supplied, $\boldsymbol{F}^{\mathrm{f}}$ and $\boldsymbol{\Delta}$ are in one-to-one correspondence.

The fractured material may, in turn, accommodate a second family of faults:

$$
\boldsymbol{F}=\boldsymbol{F}^{\mathrm{m} 1} \boldsymbol{F}^{\mathrm{f}}, \quad \boldsymbol{F}^{\mathrm{m} 1}=\boldsymbol{F}^{\mathrm{m} 2} \boldsymbol{F}^{\mathrm{f}^{2}}
$$

This decomposition can be applied recursively for as many levels as necessary; the innermost level will maintain a purely elastic behavior $\boldsymbol{F}^{\mathrm{e}}$. 


\subsection{Constitutive assumptions}

The constitutive behavior of the brittle damage model follows the introduction of a free energy density sum of two contributions with full separation of variables

$$
A\left(\boldsymbol{F}^{\mathrm{m}}, \Delta, q\right)=W^{\mathrm{m}}\left(\boldsymbol{F}^{\mathrm{m}}\right)+\frac{1}{L} \Phi(\Delta, q)
$$

where $W^{\mathrm{m}}$ is the strain-energy density per unit volume of the matrix, $\Phi$ is the cohesive energy per unit surface of faults, suitably divided by the length $L$ to provide a specific energy per unit of volume, and $q$ is a scalar internal variable used to enforce irreversibility. The particular form of the energy densities $W^{\mathrm{m}}$ and $\Phi$ can be selected freely according to the particular material considered. Note that the separation of the variables excludes strong coupling between the two energies.

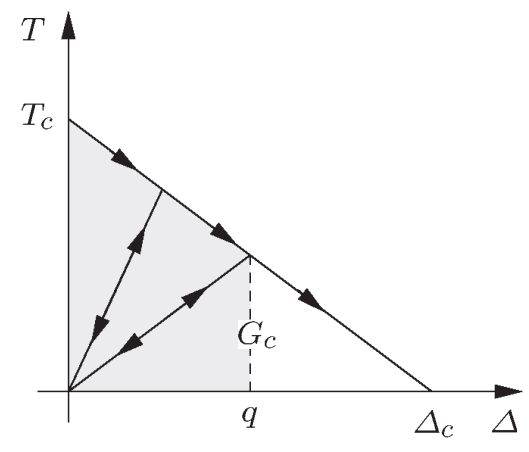

(a)

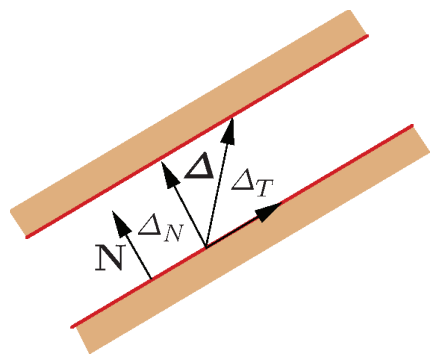

(b)

Figure 2: (a) Irreversible linear decreasing cohesive law in terms of effective opening displacement and effective traction. The enclosed area represents the critical energy release rate $G_{c}$. The maximum traction is defined by the cohesive resistance $T_{c}$, and the the maximum opening displacement is indicated by $\Delta_{c}$. The maximum attained effective opening $q$ defines the internal variable of the model, used to distinguish between first loading and unloading/reloading behaviors. (b) Kinematics of the single fault, defined by an opening displacement $\Delta$, with a component $\Delta_{N}$ along the normal and a component $\Delta_{T}$ in the plane of the fault.

The cohesive energy of a fault with orientation $N$ is assumed to depend on an effective scalar opening displacement $\Delta$ defined as

$$
\Delta=\sqrt{\left(1-\beta^{2}\right)(\Delta \cdot N)^{2}+\beta^{2}|\Delta|^{2}}
$$

where $|\Delta|$ is the norm of the opening displacement and $\beta$ a material parameter measuring the ratio between the shear and tensile strengths of the material [28]. It follows that the cohesive behavior is expressed in terms of an effective cohesive law $\Phi(\Delta, q)=\Phi(\Delta, q)$, dependent on the effective opening displacement only. The effective traction $T$ is given by

$$
T=\frac{\partial \Phi}{\partial \Delta}=\sqrt{\left(1-\beta^{-2}\right)(\boldsymbol{T} \cdot \boldsymbol{N})^{2}+\beta^{-2}|\boldsymbol{T}|^{2}}
$$

In applications, we use a simple effective cohesive law, visualized Fig. 2(a) During the first opening, the cohesive law follows a linearly decreasing envelope, i. e.,

$$
\Phi(\Delta, q)=\left\{\begin{array}{cc}
T_{c} \Delta\left(1-0.5 \Delta / \Delta_{c}\right) & \text { if } \Delta \leq \Delta_{c} \\
G_{c}=0.5 T_{c} \Delta_{c} & \text { otherwise }
\end{array},\right.
$$

where $G_{c}$ is the critical energy release rate of the material, $T_{c}$ the tensile resistance, and $\Delta_{c}$ the critical opening displacement corresponding to the full decohesion of the faults. Fracture is an irreversible process, 
thus decohered faults permanently damage the material. The extent of damage is expressed through the the maximum attained effective opening displacement $q=\Delta_{\max }$. Irreversibility is enforced by assuming unloading and reloading to/from the origin, see Fig. 2(a) according to the kinetic equation

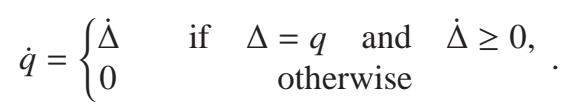

Tractions acting on the cohesive surface follows as, cf. [24],

$$
\boldsymbol{T}=\frac{\partial \Phi}{\partial \boldsymbol{\Delta}}=\frac{T}{\Delta}\left[\left(1-\beta^{2}\right)(\boldsymbol{\Delta} \cdot \boldsymbol{N}) \boldsymbol{N}+\beta^{2} \Delta\right],
$$

In the derivation of the constitutive model is necessary to introduce the configurational force conjugate to $N$, given by

$$
\frac{\partial \Phi}{\partial N}=\frac{T}{\Delta}\left(1-\beta^{2}\right)(\Delta \cdot N) \Delta
$$

Damage irreversibility is a constraint of the brittle damage model, enforced in calculations through the growth condition $\dot{q}>0$. Moreover, upon fault closure the material model has to satisfy the impenetrability constraint, i. e., the component of the opening displacement along the normal to the faults cannot be negative, thus $\Delta \cdot \boldsymbol{N} \geq 0$. More importantly, the model accounts for internal friction, a major dissipation mechanism in geological applications. We assume that friction operates at the faults concurrently with cohesion. Clearly, friction can become the sole dissipative mechanics if the faults loose cohesion completely upon the attainment of the critical opening displacement $\Delta_{c}$. In considering friction, we resort to the approach proposed in Pandolfi et al. [29] and make use of a dual dissipation potential per unit area $\Psi^{*}(\dot{\Delta} ; \boldsymbol{F}, \boldsymbol{\Delta}, q)$, where $\dot{\Delta}$ denotes the rate of the fault opening displacement.

\subsection{Variational Characterization}

The behavior of irreversible materials with friction can be characterized variationally by recourse to time discretization [30, 24], where a process of deformation is analyzed at distinct successive times $t_{0}, \ldots, t_{n+1}=$ $t_{n}+\Delta t, \ldots$ We assume that the state of the material at time $t_{n}\left(\boldsymbol{\Delta}_{n}\right.$ and $\left.q_{n}\right)$ is known and the total deformation $\boldsymbol{F}_{n+1}$ at time $t_{n+1}$ is assigned. The problem is to determine the state of the material at time $t_{n+1}$, accounting for material constraints and dissipation.

Following [30, 24], the variational characterization of the material model requires to obtain an effective, incremental, strain-energy density $W_{n}\left(\boldsymbol{F}_{n+1}\right)$ by evaluating the infimum with respect to $\boldsymbol{\Delta}_{n+1}$ and $q_{n+1}$ of the extended constrained energy defined as

$$
\begin{aligned}
W_{n}\left(\boldsymbol{F}_{n+1}\right)= & \inf _{\substack{\boldsymbol{\Delta}_{n+1}, q_{n+1}}} A\left(\boldsymbol{F}_{n+1}, \boldsymbol{\Delta}_{n+1}, q_{n+1}\right)+\frac{\Delta t}{L} \psi^{*}\left(\frac{\boldsymbol{\Delta}_{n+1}-\boldsymbol{\Delta}_{n}}{\Delta t} ; \boldsymbol{F}_{n+1}, \boldsymbol{\Delta}_{n+1}, q_{n+1}\right) . \\
& \boldsymbol{\Delta}_{n+1} \cdot \boldsymbol{N} \geq 0 \\
& q_{n+1} \geq q_{n}
\end{aligned}
$$

The subindex $n$ used in $W_{n}$ signifies the dependence on the initial state. The irreversibility and the impenetrability constraints render the effective strain-energy density $W_{n}$ dependent on the initial conditions at time $t_{n}$, and account for all the inelastic behaviors, such as damage, hysteresis, and path dependency. The constraints of the minimum problem can be enforced by means of two Lagrange multipliers $\lambda_{1}$ and $\lambda_{2}$, cf. [24]. Optimization leads to a system of four equations, that provide $\boldsymbol{\Delta}_{n+1}, q_{n+1}, \lambda_{1}$, and $\lambda_{2}$. Thus, $W_{n}\left(\boldsymbol{F}_{n+1}\right)$ acts as a potential for the first Piola-Kirchhoff stress tensor $\boldsymbol{P}_{n+1}$ at time $t_{n+1}$ [30], i. e., as

$$
\boldsymbol{P}_{n+1}=\frac{\partial W_{n}\left(\boldsymbol{F}_{n+1}\right)}{\partial \boldsymbol{F}_{n+1}} .
$$

The stable equilibrium configurations are the minimizers of the corresponding effective energy. Note that the variational formulation Eq. (9) of fault friction is non-standard in that it results in an incremental minimization problem. In particular, the tangent stiffness corresponding to the incremental equilibrium problem 
is symmetric, contrary to what is generally expected of non-associative materials. In calculations we assume rate independent Coulomb friction and, for the linearly decreasing cohesive model, we set

$$
\psi^{*}\left(\dot{\Delta} ; S^{\mathrm{m}}, \boldsymbol{N}\right)=\mu_{f} \max \left\{0,-\boldsymbol{N} \cdot \boldsymbol{S}^{\mathrm{m}} \boldsymbol{N}\right\}|\dot{\boldsymbol{\Delta}}|
$$

where $\mu_{f}$ is the coefficient of friction and we denote the symmetric second Piola-Kirchhoff stress tensor of the matrix of components

$$
S_{I J}^{\mathrm{m}}=F_{i I}^{\mathrm{m}} \frac{\partial W^{\mathrm{m}}}{\partial F_{i J}^{\mathrm{m}}}
$$

The dual dissipation potential in Eq. (11) is rate-independent, i. e., is positively homogeneous of degree 1 in $\dot{\Delta}$, and proportional to the contact pressure.

The fault geometrical features $N$ and $L$ can be determined with the aid of the time-discretized variational formulation as explained in the next section, cf. [24].

\subsection{Fault Inception and Orientation}

The actual orientation $N$ of the faults is defined by the surrounding stress state. Suppose that the material is undamaged at time $t_{n}$ and that we are given the deformation $\boldsymbol{F}_{n+1}$ at time $t_{n+1}$. We test two end states of the material, one with faults and another without faults, and choose the end state which results in the lowest incremental energy density $W_{n}\left(\boldsymbol{F}_{n+1}\right)$. The time-discretized variational formulation allows to ascertain whether the insertion of faults is energetically favorable, and the optimal orientation of the faults in the fractured material. The orientation of the faults $N$ and the remaining state variables are obtained variationally from an extended constrained minimum problem, i. e.,

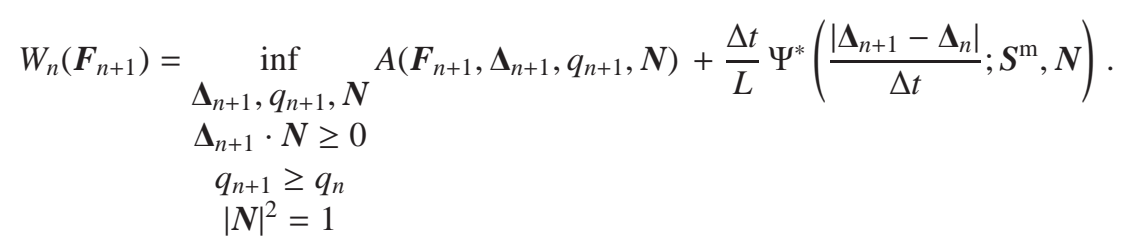

Constrained optimization leads to a set of six equations, whose solution provides the optimal orientation $N, q$, $\Delta$, and three Lagrangian multipliers. The optimal solution for faults undergoing opening without sliding has been described in [24]. For stress states in overall extension the frictional dissipation is null, and the resulting normal aligns with the direction of the maximum principal value of $\boldsymbol{S}^{\mathrm{m}}$. For stress states characterized by overall compression, here we provide a solution different from the one reported in [24], and specific for pressure sensitive materials. The two optimization equations involving the normal $N$ are (for the sake of clarity we drop the index $n+1)$ :

$$
\begin{aligned}
& \frac{\partial}{\partial \Delta_{I}}\left[A+\frac{\Delta t}{L} \Psi^{*}+\lambda_{1} \Delta \cdot N\right]=-\frac{N_{J}}{L+\Delta \cdot N} S_{J I}^{\mathrm{m}}+\frac{1}{L} \frac{\partial \Phi}{\partial \Delta_{I}}+\frac{\Delta t}{L} \frac{\partial \Psi^{*}}{\partial \Delta_{I}}+\lambda_{1} N_{I}=0 \\
& \frac{\partial}{\partial N_{I}}\left[A+\frac{\Delta t}{L} \Psi^{*}+\lambda_{1} \Delta \cdot N+\lambda_{3}|N|^{2}\right]= \\
& -\frac{\Delta_{J}}{L+\Delta \cdot N} S_{J I}^{\mathrm{m}}+\frac{1}{L} \frac{\partial \Phi}{\partial N_{I}}+\frac{\Delta t}{L} \frac{\partial \Psi^{*}}{\partial N_{I}}+\lambda_{1} \Delta_{I}+2 \lambda_{3} N_{I}=0
\end{aligned}
$$

Under a compressive stress, incipient faults are necessarily closed, $\boldsymbol{\Delta}_{n}=\underline{0}$, and can deform only by sliding, i. e., $\boldsymbol{\Delta} \cdot \boldsymbol{N}=0$. We denote with $\boldsymbol{M}=\boldsymbol{\Delta} /|\boldsymbol{\Delta}|$ the unit vector in the direction of $\boldsymbol{\Delta}$. Thus, the dissipation potential can be written as

$$
\Psi^{*}\left(\frac{\left|\Delta-\Delta_{n}\right|}{\Delta t} ; S^{\mathrm{m}}, N\right)=-\mu_{f} \boldsymbol{N} \cdot \boldsymbol{S}^{\mathrm{m}} \boldsymbol{N} \frac{|\Delta|}{\Delta t},
$$

and Eqs. 14a -14b become

$$
-S_{I J}^{\mathrm{m}} N_{J}+\beta T M_{I}-\mu_{f} \underset{9}{N} \cdot S^{\mathrm{m}} \boldsymbol{N} M_{I}+L \lambda_{1} N_{I}=0
$$




$$
-S_{I J}^{\mathrm{m}} M_{J}-2 \mu_{f} S_{J I}^{\mathrm{m}} N_{J}+L \lambda_{1} M_{I}+\frac{2 \lambda_{3} L}{|\boldsymbol{\Delta}|} N_{I}=0 .
$$

Multiplying the first of these equations by $M_{I}$ and the second by $N_{I}$ we obtain the identities

$$
\begin{gathered}
S_{I J}^{\mathrm{m}} N_{J} M_{I}+\mu_{f} S_{I J}^{\mathrm{m}} N_{J} N_{I}=\beta T \\
S_{I J}^{\mathrm{m}} N_{J} M_{I}+2 \mu_{f} S_{I J}^{\mathrm{m}} N_{J} N_{I}=\frac{2 \lambda_{3} L}{|\Delta|} .
\end{gathered}
$$

The resulting equations imply that $N$ is a plane where the matrix shear stress satisfies the Mohr-Coulomb failure criterion, in the classical form

$$
\tau=\beta T-\mu_{f} \sigma, \quad \tau=S_{I J}^{\mathrm{m}} N_{J} M_{I}, \quad \sigma=S_{I J}^{\mathrm{m}} N_{J} N_{I},
$$

where $T$ must be intended equal to $T_{c}$ at fault inception. Thus, when faults form, $\beta T_{c}$ corresponds to the cohesion (shear resistance at null normal stress) and $\mu_{f}=\tan \varphi$ the friction coefficient of the material. Eq. (17) sheds light on the meaning of the parameter $\beta$ that, for pressure sensitive materials, identifies with the friction coefficient $\mu_{f}$. Finally, Eq. (16) provides the lagrangian multiplier $\lambda_{3}$ as

$$
\lambda_{3}=\frac{|\Delta|}{2 L} \mu_{f}(T+\sigma) .
$$

Likewise, the length $L$ can be computed variationally by accounting for the misfit energy $E^{\text {mis }}(\boldsymbol{\Delta}, L)$ contained in the boundary layers that form at the junctions between faults and a confining boundary. In the model, the compatibility between the faults and their container is satisfied only on average, and this gives rise to boundary layers that penetrate into the faulted region to a certain depth. The addition to the energy furnishes a selection mechanism among all possible microstructures leading to a relaxed energy, cf. [24].

So far we have been considering either an intact material or a single family of parallel faults. The material with a single fault family is referred to as rank-1 faulting pattern material. More complex microstructures can effectively be generated by applying the previous construction recursively. In the first level of recursion, we simply replace the elastic strain-energy density $W^{\mathrm{m}}\left(\boldsymbol{F}^{\mathrm{m}}\right)$ of the matrix by $W_{n}\left(\boldsymbol{F}^{\mathrm{m}}\right)$, i. e., by the effective strainenergy density of a rank-1 faulting pattern. This substitution can now be iterated, resulting in a recursive definition of $W_{n}\left(\boldsymbol{F}_{n+1}\right)$. The recursion stops when the matrix between the faults remains elastic. The level of recursion is the rank of the microstructure. The resulting microstructures consist of faults within faults and are shown in Fig.1 (1).

According to the particular loading history, at the time $t_{n}$ and at the generic point the material is be characterized by a particular microstructure with several $\Delta$, determined in respect of equilibrium and compatibility conditions. The model is therefore able to account for variable opening of the faults.

\section{Permeability of the brittle damage model}

Permeability is an overall important physical property of porous media very difficult to characterize theoretically. For simple and structured models of porous media, permeability can be estimated through analytical relationships that apply only under a narrow range of conditions. The class of Kozeny-Carman type models collects simple relations that, under the assumption of laminar flow of the pore fluid, link the permeability to the microstructural characteristics of the porous medium. The original Kozeny-Carman relation [31, 32, 33] reads

$$
k=\frac{c}{8 a_{v}^{2} \tau} n\left(\frac{n}{1-n}\right)^{2}
$$

where $k$ is a scalar permeability, $c$ an empirical geometric parameter, $a_{v}$ the ratio of the exposed surface of the channels to the volume of the solids (also called specific internal s urface area), and $\tau$ the tortuosity, related to the ratio between $L_{a}$, average length of the channels, and $L$, macroscopic length of the flow path. 
The estimation of the shape coefficients $a_{v}$ and $\tau$ has been promoting an active research [34, 35, 36, 37, 38]. electrical conductivity of rocks and the brine (salt solution) saturation through quantitative relations [39, 40, 41, 42, 43, 44, 45]. The complexity of the relationship between the permeability tensor and a scalar property such as the porosity in rocks has been clearly pointed out [46].

The scalar nature of variables and parameters used in analytical models leads to scalar definitions, and the correct tensor nature of the permeability is disregarded. Therefore, such models are not meaningful if applied to soils characterized by the presence of sedimentation layers or fissures. Moreover, these models do not allow for the modification of the porous medium microstructure due to fluid-porous matrix interactions, or by the presence of a variable confining pressure. In particular, permeability depends not only on the actual stress and on the strain during the loading history, but also on the evolution of the crack patterns, which is anisotropic in nature.

Under the assumption of a perfectly impermeable matrix and considering the presence of a single fault family, the permeability tensor for the fractured brittle damage model can be directly derived from the particular geometry of faults. The permeability of a particular geometry of parallel and equidistant faults has been examined by Irmay [47]. Snow [25, 26] and Parsons [27] obtained expression for anisotropic permeability, similar to the one described here, by considering networks of parallel fissures.

We begin by recalling that the opening displacement decomposes into a normal $\Delta_{N}$ and a sliding $\Delta_{T}$ components, see Fig. 2(b), computed as:

$$
\Delta_{N}=N \cdot \Delta, \quad \Delta_{S}=(I-N \otimes N) \Delta, \quad \Delta_{S}=\left|\Delta_{S}\right|,
$$

Let us assume that a fluid flows within the faults, filling the open layers of constant width $\Delta_{N}$. The average fluid flow, in laminar regime, will take place in the plane of the layer. According to the solution of the Navier-Stokes' equation, the average velocity $v_{s}$ along the generic direction $s$ in the plane of the fault is

$$
v_{s}=-\frac{\Delta_{N}^{2}}{12} \frac{\rho g}{\mu} \frac{\partial h}{\partial s},
$$

where $\partial h / \partial s$ is the hydraulic head gradient in the direction $s$. The assumption of laminar flow through a crack has been widely used in the literature, cf., e. g., [13, 48]. By considering a porous medium made of several parallel faults of equal width, the discharge $q_{s}$ in the direction of the flow is

$$
q_{s}=n^{\mathrm{f}} v_{s}=-\frac{\Delta_{N}}{L+\Delta_{N}} \frac{\Delta_{N}^{2}}{12} \frac{\rho g}{\mu} \frac{\partial h}{\partial s},
$$

where

$$
n^{\mathrm{f}}=\frac{\Delta_{N}}{L+\Delta_{N}}
$$

is a measure of the porosity due exclusively to the presence of faults. By comparing Eqs. (20) and (1), we obtain the permeability of the fractured material in direction $s$ as

$$
k_{s}=\frac{\Delta_{N}}{L+\Delta_{N}} \frac{\Delta_{N}^{2}}{12} .
$$

Now we want to restate the above equations in vector form. The hydraulic gradient $\boldsymbol{\nabla} h$ and the unit vector $\boldsymbol{d}$ in the direction of the fluid flow are, respectively,

$$
\boldsymbol{\nabla} h=\frac{\partial h}{\partial x_{1}} \boldsymbol{e}_{1}+\frac{\partial h}{\partial x_{2}} \boldsymbol{e}_{2}+\frac{\partial h}{\partial x_{3}} \boldsymbol{e}_{3}, \quad \boldsymbol{d}=\frac{\partial x_{1}}{\partial s} \boldsymbol{e}_{1}+\frac{\partial x_{2}}{\partial s} \boldsymbol{e}_{2}+\frac{\partial x_{3}}{\partial s} \boldsymbol{e}_{3} .
$$

Thus, the directional gradient $\partial h / \partial s$ can be expressed as

$$
\frac{\partial h}{\partial s}=\nabla h \cdot \boldsymbol{d}=\frac{\partial h}{\partial x_{1}} \frac{\partial x_{1}}{\partial s}+\frac{\partial h}{\partial x_{2}} \frac{\partial x_{2}}{\partial s}+\frac{\partial h}{\partial x_{3}} \frac{\partial x_{3}}{\partial s} .
$$


The average velocity $v_{s}$ in Eq. 19 can be written as

$$
v_{s}=-\frac{\Delta_{N}^{2}}{12} \frac{\rho g}{\mu} \nabla h \cdot d
$$

and the average flow velocity vector, $\boldsymbol{v}_{s}=v_{s} \boldsymbol{d}$, becomes

$$
\boldsymbol{v}_{s}=-\frac{\Delta_{N}^{2}}{12} \frac{\rho g}{\mu}(\nabla h \cdot d) d .
$$

Now, the hydraulic discharge can be written as

$$
\boldsymbol{q}_{s}=n^{\mathrm{f}} \boldsymbol{v}_{s}=-\frac{\Delta_{N}}{L+\Delta_{N}} \frac{\Delta_{N}^{2}}{12}(\boldsymbol{d} \otimes \boldsymbol{d}) \frac{\rho g}{\mu} \nabla h,
$$

thus the permeability tensor due to the presence of the faults $\boldsymbol{k}^{\mathrm{f}}$ derives as

$$
\boldsymbol{k}^{\mathrm{f}}=\frac{\Delta_{N}}{L+\Delta_{N}} \frac{\Delta_{N}^{2}}{12}(\boldsymbol{d} \otimes \boldsymbol{d})
$$

To account for a generic direction of the flow in the layer of normal $N$, in Eq. (28) we must replace the unit vector $\boldsymbol{d}$ with the projection $(\boldsymbol{I}-\boldsymbol{N} \otimes \boldsymbol{N})$, reaching the expression

$$
\boldsymbol{k}^{\mathrm{f}}=\frac{\Delta_{N}}{L+\Delta_{N}} \frac{\Delta_{N}^{2}}{12}(\boldsymbol{I}-\boldsymbol{N} \otimes \boldsymbol{N})
$$

It follows that, as a noteworthy feature of the brittle damage model, the permeability is described by an anisotropic tensor.

If $Q$ fault families are present in the porous medium, each characterized by a normal $N^{K}$, a separation $L^{K}$, and a normal opening displacement $\Delta_{N}^{K}$, the equivalent permeability is given by the sum of the corresponding permeabilities:

$$
\boldsymbol{k}^{\mathrm{f}}=\sum_{K=1}^{Q} \frac{\Delta_{N}^{K}}{L^{K}+\Delta_{N}^{K}} \frac{\Delta_{N}^{K^{2}}}{12}\left(\boldsymbol{I}-\boldsymbol{N}^{K} \otimes \boldsymbol{N}^{K}\right) .
$$

The model does not exclude the presence of an initial porosity $n^{\mathrm{m}}$, see Eq. (2), and permeability $\boldsymbol{k}^{\mathrm{m}}$, see Eq. 177, of the intact matrix. In this case, the resulting porosity and permeability will be given by the sum of the terms corresponding to the intact matrix and to the faults

$$
n=n^{\mathrm{m}}+n^{\mathrm{f}}, \quad \boldsymbol{k}=\boldsymbol{k}^{\mathrm{m}}+\boldsymbol{k}^{\mathrm{f}} .
$$

In practical applications we assume an isotropic matrix permeability of Kozeny-Carman type, with the simplified form

$$
\boldsymbol{k}^{\mathrm{m}}=k_{\mathrm{KC}} \boldsymbol{I}, \quad k_{\mathrm{KC}}=C_{\mathrm{KC}} \frac{\left(n^{\mathrm{m}}\right)^{3}}{\left(1-n^{\mathrm{m}}\right)^{2}},
$$

where the constant $C_{\mathrm{KC}}$ accounts for shape coefficients.

We observe that the hydraulic behavior of the brittle damage model is dependent on fracture orientation and spacing computed on the basis of the boundary conditions, and that its permeability can vary according to the kinematics of the faults.

\section{Verification and Validation}

Next, we present selected examples of application of the porous damage model, starting from a simple one-dimensional analytical model that accounts for the presence of the pore pressure. We continue by showing the response of the fully tridimensional dry model undergoing a loading that mimics a hydraulic fracturing 
process. We conclude with the validation of the model, reproducing a few representative experimental results on granite and sandstone.

We note that numerical calculations of the dynamic multiaxial compression experiments on sintered aluminum nitride (AIN) of Chen and Ravichandran [49, 50, 51, 52] were presented in [24] by way of validation of the dry mechanical aspects of the model. The model was shown to correctly predict the general trends regarding the experimental observed damage patterns, as well as the brittle-to-ductile transition resulting under increasing confinement. Therefore, in the present work we restrict validation to the hydro-mechanical aspects of the model.

\subsection{Illustrative one-dimensional model with pore pressure}
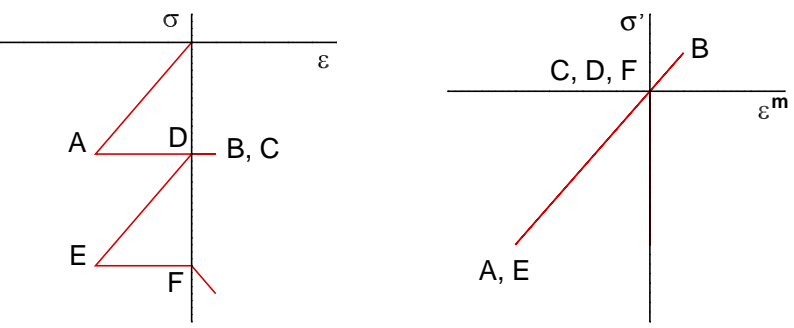

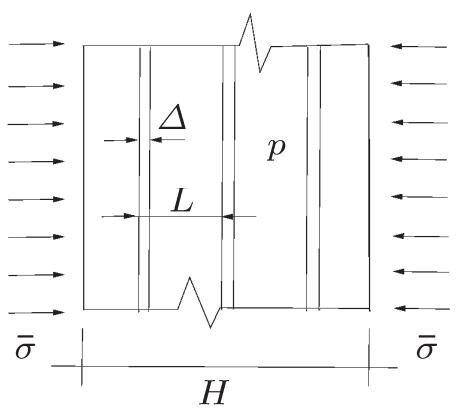

(a) Geometry
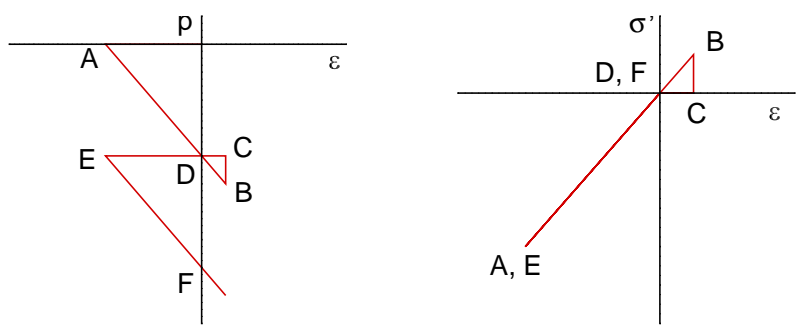

(b) Results

Figure 3: One Dimensional example. (a) Size of the slab and of the faults and external tractions. Plots of the behavior of the one dimensional example. (top left) Relationship between total stress and total strain. (top right) Relationship between effective stress and matrix strain. (bottom left) Relationship between pore pressure and total strain. (bottom right) Relationship between effective stress and total strain. Point A denotes the maximum compression in the intact material. Point B indicates the critical unstable fault inception state following the increase of pore pressure and total deformation. Point $\mathrm{C}$ indicates the equilibrium state after fault formation, with pore pressure equal to the external load. Point $\mathrm{D}$ indicates the fault reclosure induced by a reduction of the total deformation at constant pore pressure. Point $\mathrm{E}$ indicates the maximum reduction of the total deformation at constant pore pressure. Point $\mathrm{F}$ indicates the attainment of zero total deformation due to pore pressure increase.

In order to understand how the behavior of the brittle damage model in combination with the pore pressure, we begin by illustrating a uniaxial case, i. e., the infinite slab of thickness $H$ shown in Fig. 3 using a linearized version of the model equations. Tensile strains and stresses are considered positive. The material is assumed to be initially linear elastic, with no faults. The matrix is characterized by a Young modulus $E$ and a tensile resistance $T_{c}$. The slab is subject to a uniform uniaxial stress $\sigma$ due to a compressive traction $t$ acting on the boundary. If no body forces are considered, the equilibrium equation becomes

$$
\frac{d \sigma}{d x}=0, \quad \sigma=\text { const } .
$$


Terzaghi's effective stress principle states that the total stress $\sigma$ is balanced by an effective stress $\sigma^{\prime}$ and by a pore pressure $p$ acting everywhere in the porous medium, i. e.,

$$
\sigma=\sigma^{\prime}+p
$$

Following the kinematics of the brittle damage model, the total deformation $\varepsilon$ splits in elastic part, related to the behavior of the matrix, and in inelastic part, related to the presence of the faults

$$
\varepsilon=\varepsilon^{\mathrm{m}}+\varepsilon^{\mathrm{f}} .
$$

The elastic deformation defines the matrix elastic constitutive behavior in terms of effective stress, valid up to the attainment of the tensile resistance $T_{c}$

$$
\sigma^{\prime}=E \varepsilon^{\mathrm{m}}, \quad \sigma^{\prime} \leq T_{c} .
$$

If the effective stress exceeds $T_{c}$, parallel faults form at the assigned distance $L<H$, originating a discontinuous deformation $\varepsilon^{\mathrm{f}}$ due to the jump $\Delta$ of the faults

$$
\varepsilon^{\mathrm{f}}=\frac{\Delta}{L}
$$

The four equations (32)-32 involve the seven variables $\sigma, \sigma^{\prime}, p, \varepsilon, \varepsilon^{\mathrm{m}}, \varepsilon^{\mathrm{f}}$, and $\Delta$. Three variables can be assigned, the other four follow from the equations.

Let us begin by applying a growing compressive traction $t$ on the slab surfaces up to the value $t=-\bar{\sigma}$ with a null pore pressure (from the origin to the point $\mathrm{A}$ in Fig $3(\mathrm{~b})$. In this phase, we assign the total stress $\sigma, p=0$ and, since there are no faults, $\Delta=0$. We have

$$
\sigma^{\prime}=-\bar{\sigma}, \quad \varepsilon^{\mathrm{m}}=\sigma^{\prime} / E, \quad \varepsilon^{\mathrm{f}}=0, \quad \varepsilon=\varepsilon^{\mathrm{m}} .
$$

Next, we keep $\sigma=-\bar{\sigma}$ and $\Delta=0$, and let the pressure $p$ growing up to $p_{\max }=-\bar{\sigma}-T_{c}$. The matrix behaves elastically, from point A to point B in Fig 3(b), according to

$$
\sigma^{\prime}=-\bar{\sigma}-p, \quad \varepsilon^{\mathrm{m}}=\sigma^{\prime} / E, \quad \varepsilon^{\mathrm{f}}=0, \quad \varepsilon=\varepsilon^{\mathrm{m}}
$$

The stress and deformation in the matrix are compressive for small values of the pore pressure and become tensile for $p \geq-\bar{\sigma}$. Since in this phase the total deformation coincides with the elastic one, the slab expands progressively. When the pressure reaches $p_{\max }$, the effective stress reaches the resistance of the matrix, point $\mathrm{B}$ in Fig 3(b), as

$$
\sigma^{\prime}=T_{c}>0, \quad \varepsilon=\varepsilon^{\mathrm{m}}=T_{c} / E>0,
$$

and faults form at distance $L$. After failure, the presence of faults modifies the mechanics of the system. In particular, the matrix is not able to provide a stress and the matrix deformation goes to zero, i. e., $\sigma^{\prime}=0$ and $\varepsilon^{\mathrm{m}}=0$. To reach equilibrium, it is necessary to decrease the pore pressure to $p=-\bar{\sigma}$ or, alternatively, to increase the external confinement to $\bar{\sigma}$ to reach the value of $p_{\max }$. In both cases, the total deformation will be related only to the fault opening, no contribution derives from the matrix. Up to the closure of the faults, the governing equations become three, with five variables $\sigma, p \Delta, \varepsilon$ and $\varepsilon^{\mathrm{f}}$ :

$$
\sigma=p, \quad \varepsilon^{\mathrm{f}}=\varepsilon, \quad \Delta=L \varepsilon^{\mathrm{f}} .
$$

We can assign two variables and use the three equations to determine the others. For example, by keeping the total deformation constant and setting, e. g., $p=-\bar{\sigma}$, we observe the instantaneous transfer of the deformation from the matrix to the faults, point $\mathrm{C}$ in Fig $3(\mathrm{~b})$.

$$
\sigma=p=-\bar{\sigma}, \quad \varepsilon=T_{c} / E, \quad \Delta=T_{c} L / E .
$$


If we keep the extensional total deformation constant, relations (32) hold for any value of the pore pressure, because kinematics and equilibrium are not related by constitutive assumptions, and changing $p$ or $\sigma$ will have no effect on $\Delta$. The behavior of the system can be further investigated by controlling the total deformation $\varepsilon$. Let us keep the pore pressure constant, $p=-\bar{\sigma}$, and modify the boundary condition by progressively reducing $\varepsilon$. Until the total deformation is positive, $0 \leq \varepsilon \leq T_{c} / E$, faults are open and $\varepsilon^{\mathrm{m}}=0, \sigma^{\prime}=0$, from point $\mathrm{C}$ to point $\mathrm{D}$ in $\mathrm{Fig} 3(\mathrm{~b})$, The opening displacement derives from compatibility as

$$
\sigma=p, \quad \Delta=\varepsilon L .
$$

When the total deformation vanishes, faults close and enter in contact with $\Delta=0$, and the constitutive relation in the matrix is reactivated. For $\varepsilon<0$ the total deformation transfers to the matrix, $\varepsilon=\varepsilon^{\mathrm{m}}$, restoring a compressive effective stress

$$
\sigma^{\prime}=E \varepsilon^{\mathrm{m}}, \quad \sigma=E \varepsilon^{\mathrm{m}}+p .
$$

The compressive total stress increases proportionally to the total deformation, from point $\mathrm{D}$ to point $\mathrm{E}$ in Fig 3(b). Then, if we set the total stress constant, the total deformation can be progressively recovered by increasing the pore pressure, form point $\mathrm{E}$ to point $\mathrm{F}$ in Fig 3(b),

\subsection{Illustrative dry example}

The next examples are conducted in finite kinematics. We specialize the strain energy density $W$ to a neo-Hookean material extended to the compressible range, i. e.,

$$
W\left(\boldsymbol{F}^{\mathrm{m}}\right)=\frac{1}{2} \lambda \log ^{2} J^{\mathrm{m}}+\frac{1}{2} G\left(\left(\boldsymbol{F}^{\mathrm{m} T} \boldsymbol{F}^{\mathrm{m}}\right): \boldsymbol{I}-3-2 \log J^{\mathrm{m}}\right)
$$

where $\lambda$ and $G$ are the Lamé coefficients, and $J^{\mathrm{m}}=\operatorname{det} \boldsymbol{F}^{\mathrm{m}}$ is the determinant of $\boldsymbol{F}^{\mathrm{m}}$. We study the response of the brittle damage model to the action of external loadings mimicking the in-field conditions observed during hydraulic fracturing procedures, and analyze the correspondent variation in permeability. We assume an intact material, with no pre-existent or natural faults, and limit our attention to the constitutive behavior. The material is characterized by the constants listed in Table 2 The porosity and the permeability of the

Table 2: Rock material constants adopted in the illustrative examples

\begin{tabular}{ccccccc}
\hline$\lambda(\mathrm{MPa})$ & $G(\mathrm{MPa})$ & $T_{c}(\mathrm{MPa})$ & $G_{c}(\mathrm{~N} / \mathrm{mm})$ & $\varphi$ & $k_{0}$ & $n_{0}$ \\
\hline 2778 & 4167 & 10.0 & 0.1 & 45.0 & 0 & 0 \\
\hline
\end{tabular}

intact matrix are assumed to be null, thus the permeability will be exclusively related to the formations of faults. The material is allowed to form up to three families of faults, with different orientation $N^{K}$. Tensile stresses and deformations are considered positive.

By assigning a prescribed history to the deformation gradient, we simulate a multistage multiaxial test that mimics the in-field variation in stress and permeability due to hydraulic fracture. The material is initially compressed isotropically by applying a uniform stretch $L / L_{0}=\lambda_{1}=\lambda_{2}=\lambda_{3}=0.99$, to induce a geostaticlike stress state. Then, the material undergoes an isotropic extension $\lambda_{1}=\lambda_{2}=\lambda_{3}=1.01$, associated to the reduction of the effective stress due to the injection of a high pressure fracturing fluid. Given the isotropy of the stress state, at the extension corresponding to the attainment material strength the material fails in tension, creating in sequence three families of faults, with normal in the directions $\boldsymbol{e}_{1}, \boldsymbol{e}_{2}$, and $\boldsymbol{e}_{3}$, respectively. The microstructure of the three families differs because of different spacings. Upon fault closure, the failed material is able to sustain an overall compressive stress, the interpenetration of the faults being controlled by the contact algorithm. In the last stage of loading, the material is compressed with an anisotropic stretch. A $\lambda_{1}=\lambda_{2}=0.97$ stretch is applied in direction $\boldsymbol{e}_{1}$ and $\boldsymbol{e}_{2}$, while the original geostatic-like stretch $\lambda_{3}=0.99$ is applied in direction $\boldsymbol{e}_{3}$. Fig. (4) a) shows the mechanical response of the model in direction $\boldsymbol{e}_{1}$. The material initially undergoes a compression (black circles). The following extension induces a tensile state that reaches 


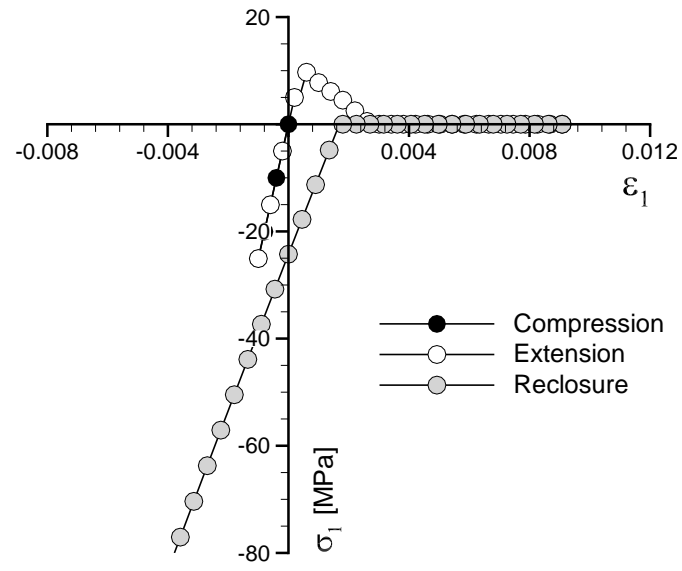

(a) Stress-Strain

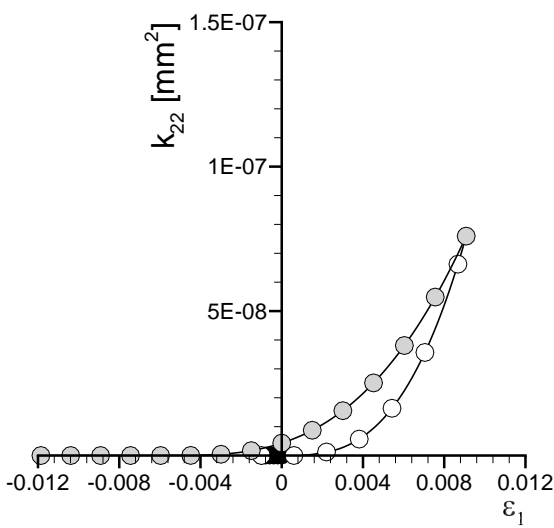

(c) Permeability $k_{22}$

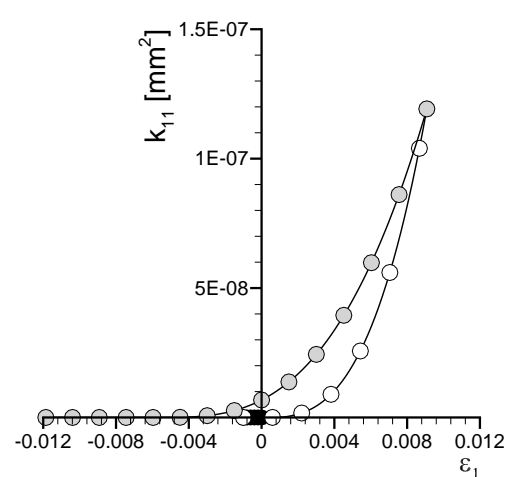

(b) Permeability $k_{11}$

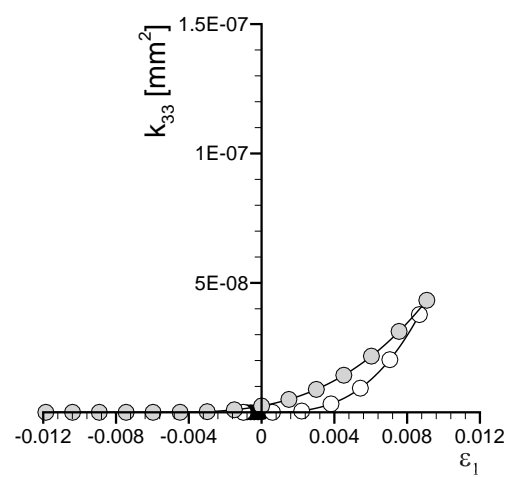

(d) Permeability $k_{33}$

Figure 4: Multi-stage multiaxial response of the material, undergoing an isotropic compression, followed by an isotropic extension, and by a final anisotropic compression. (a) Stress $\sigma_{1}(\mathrm{MPa})$ vs strain $\varepsilon_{1}$. Although the material fails, generating three nested families of faults, the material preserves its ability of sustaining load. (b) Permeability $\left(\mathrm{mm}^{2}\right)$ in direction $\boldsymbol{e}_{1}$ as a function of the strain. (c) Permeability $\left(\mathrm{mm}^{2}\right)$ in direction $\boldsymbol{e}_{2}$ as a function of the strain. (d) Permeability $\left(\mathrm{mm}^{2}\right)$ in direction $\boldsymbol{e}_{3}$ as a function of the strain.

the strength of the material and causes triple tensile failure (open circles). The final compressive stretch is characterized by a null stress until faults close completely. Afterwards, the contact algorithm provides the compressive tractions that guarantee the equilibrium of the system (grey circles). The resulting reduction of the stiffness of the material due to the damage is remarkable.

Figs. 4 (b-d) show the permeability in direction $\boldsymbol{e}_{1}, \boldsymbol{e}_{2}$, and $\boldsymbol{e}_{3}$, respectively. The permeability is null until the material fails (black circles). Then the permeability reaches a maximum corresponding to the maximum extension imposed to the material (open circles). The different values of the maximum permeability for the three directions is the combined result of the different spacing of the fault families and of the stress anisotropy derived from the formation of faults. Upon fault closure, permeability decreases to zero (gray circles). Note 
that the anisotropy of the compressive loading causes anisotropy in the permeability history. In particular, the permeability reduces more quickly in the direction $\boldsymbol{e}_{3}$, where no extra-confinement is applied. In fact, the over-compression in the two directions $\boldsymbol{e}_{1}$ and $\boldsymbol{e}_{2}$ closes the faults parallel to direction $\boldsymbol{e}_{3}$, while the flow is still allowed in the faults normal to $\boldsymbol{e}_{3}$.

\subsection{Validation of the porous model against experimental results}

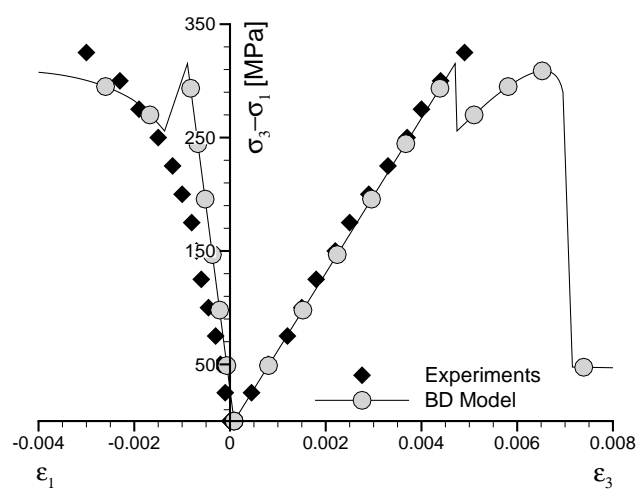

(a) Lac du Bonnet granite stress-strain

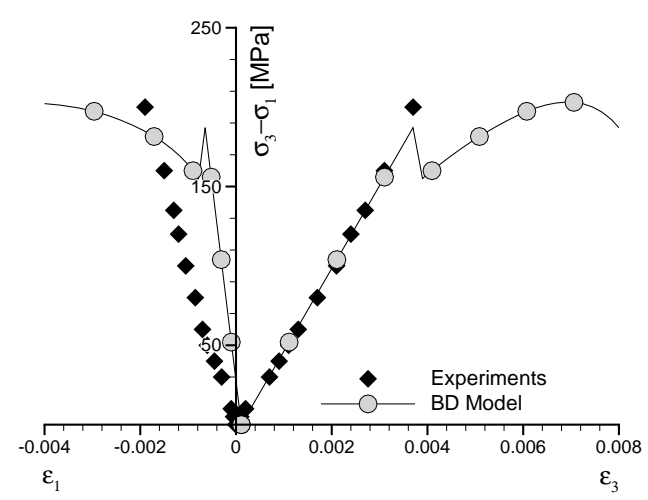

(c) Beishan granite stress-strain

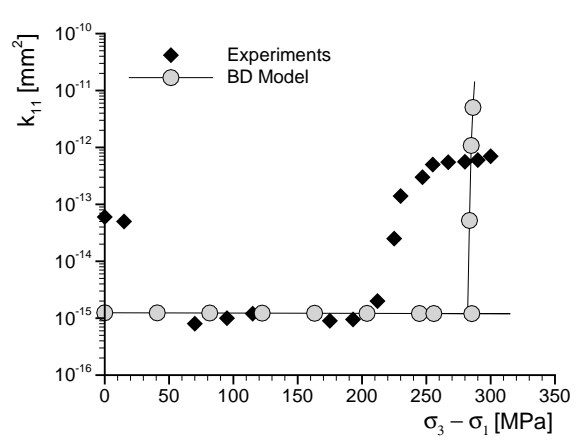

(b) Lac du Bonnet granite permeability

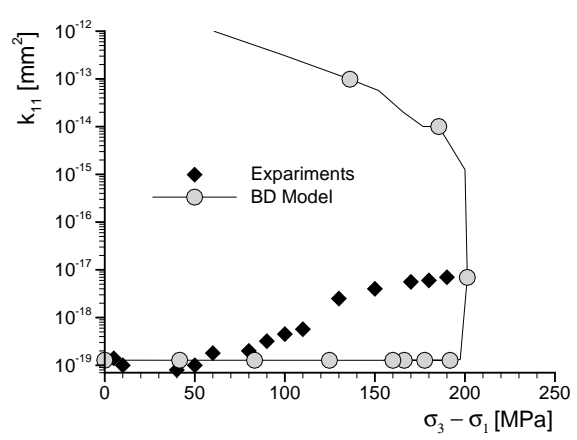

(d) Beishan granite permeability

Figure 5: Validation of the brittle damage model versus the experimental tests on Lac du Bonnet [18] and Beisahn [53] granites . The sample is confined with a $10 \mathrm{MPa}$ pressure. (a)-(c) Deviatoric stress - strain behavior. (b)-(d) Permeability variation with the deviatoric stress.

Experimental data of triaxial compression tests with permeability measurements are available form the literature. In this section we validate the porous brittle damage model, considered at the constitutive level, against experimental results of tests performed on different materials. To facilitate the comparison with the experiments and according to the typical conventions of geomechanics, in this section we assume compressive strains and stresses as positive. Material constants of the model used in the validation are listed in Table 3 . Elastic modulus $E$, Poisson coefficient $v$, friction coefficient $\mu_{f}$, porosity $n_{0}$ and permeability $k_{0}$ of the intact rocks were recovered from the experimental papers. From $E$ and $v$ we computed the Lamé constants needed by the elasticity model through the relations

$$
\lambda=\frac{v E}{(1+v)(1-2 v)}, \quad G=\frac{E}{2(1+v)} .
$$


Table 3: Material constants used for the validation of the material model

\begin{tabular}{cccccccc}
\hline Rock & $E(\mathrm{MPa})$ & $v$ & $T_{c}(\mathrm{MPa})$ & $G_{c}(\mathrm{~N} / \mathrm{mm})$ & $\varphi\left(^{\circ}\right)$ & $k_{0}\left(\mathrm{~mm}^{2}\right)$ & $n_{0}$ \\
\hline Lac du Bonnet granite [18] & 68,000 & 0.21 & 50 & 10 & 46.4 & $10^{-13}$ & 0.20 \\
Beisahn granite [53] & 52,000 & 0.21 & 60 & 10 & 35.0 & $10^{-20}$ & 0.08 \\
Berea sandstone [54] & 8,000 & 0.18 & 50 & 50 & 29.0 & $10^{-5}$ & 0.21 \\
\hline
\end{tabular}

The cohesive parameters $T_{c}, G_{c}$, not available from the experimental papers, have been calibrated through preliminary analyses.

We begin with the simulation of the triaxial tests on samples of Lac di Bonnet and Beishan granites documented in [18, 53]. The tests consisted of the application of a confining pressure of $10 \mathrm{MPa}$, followed by an axial compressive load up to failure. Experiments included the measurement of the permeability of the samples, limited to the pre-peak phase. We simulate the triaxial test with the brittle damage model and compare our numerical results with experiments. Fig. 5 shows the deviatoric stress, $\sigma_{3}-\sigma_{1}$ versus axial and lateral deformations, $\varepsilon_{3}$ and $\varepsilon_{1}$, respectively, and the permeability versus deviatoric stress. During the simulated axial compression, both granites develop one family of faults in shear. The failure plane of the faults corresponds to the one predicted by the Mohr-Coulomb criterion, inclined of an angle $\pi / 4-\varphi / 2$ with respect to direction of maximum stress $\left(21.8^{\circ}\right.$ for Lac du Bonnet and $27.5^{\circ}$ for Beishan $)$. The peak of resistance corresponds to the experimental values, but the brittle damage model predicts a post-peak behavior which is not available in the experimental papers. Experiments show an initial reduction of the permeability, followed by a marked increase when the samples begin to show a reduction of stiffness. By contrast, the brittle damage model predicts a constant permeability, which does not increase even after the formation of the shear faults. However, when the load becomes too high to be balanced by friction and the axial loading reduces, faults open and the permeability increases, showing a characteristic behavior often reported in experimental literature, cf. [55] and the numerous references therein.

A second set of triaxial experiments on Berea sandstone with different confinement are reported in [54]. We selected three small confinement triaxial experiments, characterized by a softening stress-strain curve. Pre and post-peak porosity and permeability data are included in the experimental paper. We simulated the experimental tests at confining pressures of 5, 10 and $40 \mathrm{MPa}$. Experimental and numerical results are shown in Fig. 6 Fig. 6(a) shows the deviatoric stress versus the axial deformation. Simulations capture nicely the peak stress for the three tests, while the softening branch is not perfectly reproduced. Fig. 6(b) compares numerical and experimental porosity for the two tests at lower confinement pressure. In both simulation and experiment, porosity reduces progressively until the stress peak is reached, and grows during the softening phase, in correspondence to the reduction of the deviatoric stress. Simulations predict qualitatively and quantitatively the variation of porosity during the test. Comparisons between the model predictions and the experimental observations in terms of permeability are not good. In the experiments permeability decreases markedly after the stress peak, see Fig. 6(c), with a trend in contrast to the one normally observed in experimental data, cf., e. g., [55]. Indeed, in most documented experiments, permeability grows after the failure of the sample. The brittle damage model predicts a post-peak increase in permeability, see Fig. 6(d), which is opposite to the sandstone experiments, but in line with many experimental results in sandstone and other materials, and is also in agreement with the simulations on granites discussed here.

\section{Conclusions}

We have developed a model of distributed fracturing of rock masses, and the attendant permeability enhancement thereof, based on an explicit micromechanical construction resulting in complex connected patterns of cracks, or faults. The approach extends the multi-scale brittle damage material model introduced in [24], which was limited to mechanical damage. The fracture patterns that form the basis of the theory are not implied but explicitly defined and the rock mass undergoes throughout compatible deformations and 


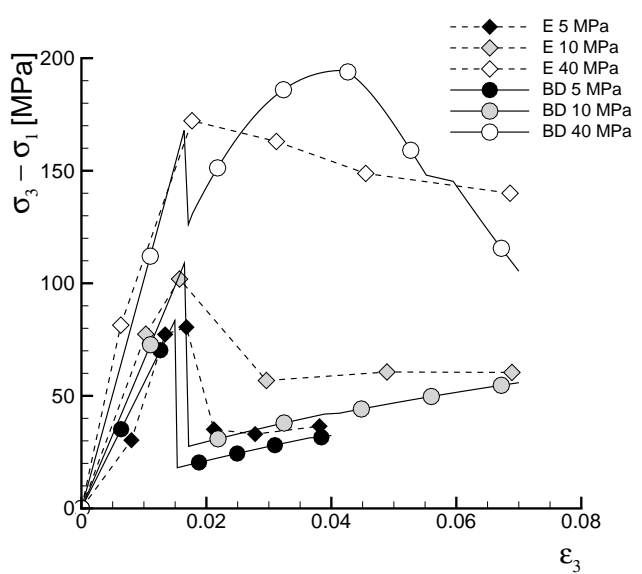

(a) Stress-Strain

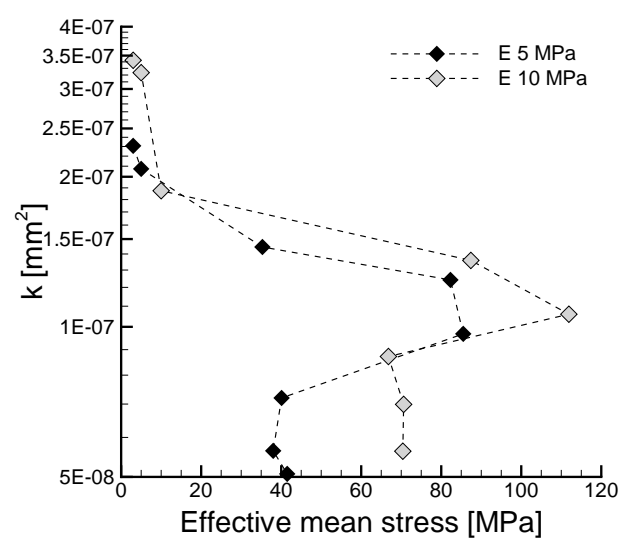

(c) Stress-Strain

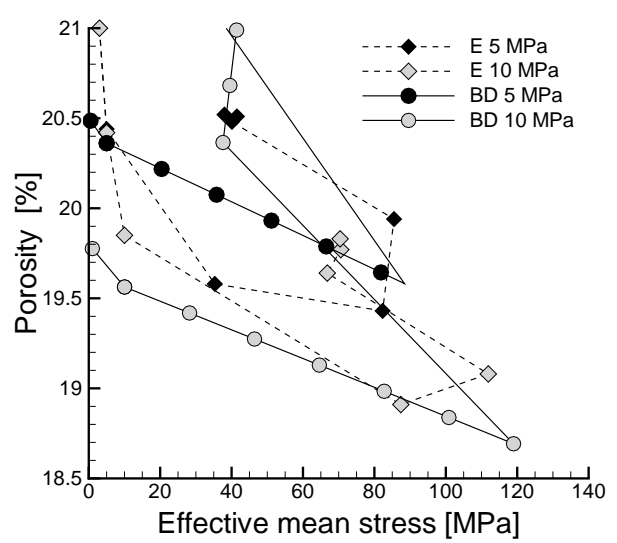

(b) Permeability

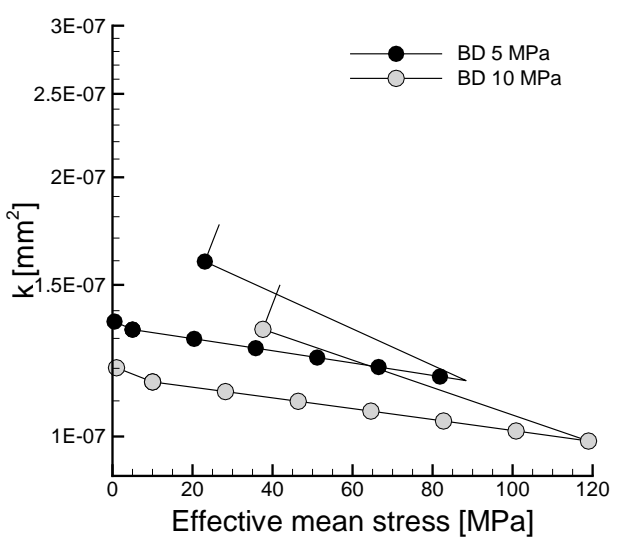

(d) Permeability

Figure 6: Validation of the brittle damage model versus the experimental tests on Berea sandstone [54]. Sample are confined with a 5, 10 or $40 \mathrm{MPa}$ pressure. (a) Deviatoric stress-axial strain behavior. (b) Porosity variation with the deviatoric stress. (c) Experimental permeability, variation with the deviatoric stress. (d) Numerical Permeability, variation with the deviatoric stress.

remains in static equilibrium, not just on average at the macroscopic scale, but also the micromechanical level. The sequential faulting construction used to generate the fracture patterns has been shown in [24] to be optimal as regards the ability of the fracture patterns to relieve stress. In addition, the nucleation criterion, orientation and spacing of the faults derive rigorously from energetic considerations. Following nucleation, fractures can deform by frictional sliding or undergo opening, thereby partially relieving the geostatic stresses in the rock mass. The extension of the theory presented in this paper additionally accounts for fluid pressure by recourse to Terzaghi's effective stress principle. Specifically, we estimate the permeability enhancement resulting from fracture enhancement using standard lubrication theory [25, 26, 27]. This extension gives rise to a fully-coupled hydro-mechanical model.

The formulation has been derived in finite kinematics to be consistent with the formulation of the damage model in [18]. A finite kinematics approach is able to describe both large and small strains, so that the model can be applied also to porous media different from rocks. A linear version of the model is currently under development, in view of heavy numerical applications in field problems. 
The dry mechanical aspects of the model were validated in [24] by means of comparisons with the dynamic multiaxial compression experiments on sintered aluminum nitride (AlN) of Chen and Ravichandran [49, 50, 51, 52]. The model was shown to correctly predict the general trends regarding the experimental observed damage patterns, as well as the brittle-to-ductile transition resulting under increasing confinement. The hydro-mechanical coupled model has been validated against three different sets of experimental data concerned with triaxial tests at different confinement pressure on granite and sandstone, including Lac du Bonnet [18] and Beisahn [53] granites and Berea sandstone [54]. The ability of the model to qualitatively reproduce the experimental peak strength, post-peak stress-strain behavior permeability enhancement during loading and recovery during unloading is remarkable.

The present coupled hydro-mechanical model has potential for use in applications, such as rocks under geostatic conditions, gravity dams, hydraulic fracture operations, and others, in which a solid deforms and undergoes extensive fracture under all-around confinement while simultaneously being infiltrated by a fluid. The particular case of hydraulic fracture is characterized by the injection of fluid at high pressure, which actively promotes the fracture process and the transport of fluid into the rock mass. Under such conditions, the present model is expected to predict the development of three-dimensional fracture patterns of great complexity over multiple scales. Such complex fracture patterns have indeed been inferred from acoustic measurements in actual hydraulic fracture operations [56, 57] and are in sharp contrast to traditional models of hydraulic fracture, which posit the formation of a single mathematically-sharp crack. The present model thus represents a paradigm shift from said traditional models in its ability to account for complexity in the fracture pattern over multiple scale while simultaneously supplying macroscopic effective properties such as permeability and strength that can in turn be used, e. g., in full-field finite element simulations.

\section{Acknowledgments}

MLDB, GDV, and AP wish to acknowledge the financial support of the Ente Nazionale Idrocarburi San Donato Milanese, Italy, under the research contract \#3500034224.

\section{Appendix A}

By denoting the time derivative with $\dot{n}$, we write

$$
V n=V_{V}, \quad \dot{V} n+V \dot{n}=\dot{V}_{V} \quad \dot{n}=\frac{\dot{V}_{V}}{V}-\frac{\dot{V}}{V} n .
$$

Under the assumption of solid particle incompressibility, $\dot{V}=\dot{V}_{v}$ and $\dot{J}=\dot{V}_{v} / V_{0}$ thus the rate of porosity change becomes:

$$
\dot{n}=(1-n) \frac{\dot{V}_{V}}{V_{0}} \frac{V_{0}}{V}=(1-n) \frac{\dot{J}}{J} .
$$

This relation can be alternatively written in the form

$$
\frac{\dot{n}}{1-n}=\frac{\dot{J}}{J}, \quad-\log (1-n)=\log J+C,
$$

where $C$ is a constant, which can be derived by setting as initial values $J_{0}=1$ and $n_{0}$, obtaining

$$
n=1+\frac{1}{J}\left(n_{0}-1\right)
$$




\section{References}

[1] B. B. S. Singhal and R. P. Gupta. Applied Hydrogeology of Fractured Rocks. Springer Science \& Business Media, 2010.

[2] A. Aydin. Fractures, faults, and hydrocarbon entrapment, migration and flow. Marine and Petroleum Geology, 17(7):797-814, 2000.

[3] Y. L. Lu, D. Elsworth, and L. G. Wang. Microcrack-based coupled damage and flow modeling of fracturing evolution in permeable brittle rocks. Computers and Geotechnics, 49:226-244, 2013.

[4] S. Levasseur, F. Collin, R. Charlier, and D. Kondo. A micro-macro approach of permeability evolution in rocks excavation damaged zones. Computers and Geotechnics, 49:245-252, 2013.

[5] R. H. C. Wong, K. T. Chau, and P. Wang. Microcracking and grain size effect in yuen long marbles. International Journal of Rock Mechanics and Mining Sciences, 33(5):479-485, 1996.

[6] R. L. Kranz. Microcracks in rocks: a review. Tectonophysics, 100(1):449-480, 1983.

[7] Z. P. Bažant, M. Salviato, V. T. Chau, H. Viswanathan, and A. Zubelewicz. Why fracking works. Journal of Applied Mechanics, 81(10):101010, 2014.

[8] S. C. Yuan and J. P. Harrison. A review of the state of the art in modelling progressive mechanical breakdown and associated fluid flow in intact heterogeneous rocks. International Journal of Rock Mechanics and Mining Sciences, 43(7):1001-1022, 2006.

[9] R. W. Zimmerman. Effective conductivity of a two-dimensional medium containing elliptical inhomogeneities. In Proceedings of the Royal Society of London A: Mathematical, Physical and Engineering Sciences, volume 452, pages 1713-1727. The Royal Society, 1996.

[10] V. Mityushev and P. M. Adler. Darcy flow around a two-dimensional permeable lens. Journal of Physics A: Mathematical and General, 39(14):3545, 2006.

[11] L. Dormieux and D. Kondo. Approche micromécanique du couplage perméabilité-endommagement. Comptes Rendus Mécanique, 332(2):135-140, 2004.

[12] A. Pouya. Three-dimensional flow in fractured porous media: a potential solution based on singular integral equations. Advances in Water Resources, 35:30-40, 2012.

[13] A. Pouya and M.-N. Vu. Numerical modelling of steady-state flow in $2 \mathrm{~d}$ cracked anisotropic porous media by singular integral equations method. Transport in porous media, 93(3):475-493, 2012.

[14] M.-N. Vu, A. Pouya, and D. M. Seyedi. Modelling of steady-state fluid flow in 3d fractured isotropic porous media: application to effective permeability calculation. International Journal for Numerical and Analytical Methods in Geomechanics, 37(14):2257$2277,2013$.

[15] M. Oda. Permeability tensor for discontinuous rock masses. Géotechnique, 35(4):483-495, 1985.

[16] C. A. Tang, L. G. Tham, P. K. K. Lee, T. H. Yang, and L. C. Li. Coupled analysis of flow, stress and damage (fsd) in rock failure. International Journal of Rock Mechanics and Mining Sciences, 39(4):477-489, 2002.

[17] F. Homand-Etienne, D. Hoxha, and J.-F. Shao. A continuum damage constitutive law for brittle rocks. Computers and Geotechnics, 22(2):135-151, 1998.

[18] M. Souley, F. Homand, S. Pepa, and D. Hoxha. Damage induced permeability changes in granite: a case example at the URL in Canada. International Journal of Rock Mechanics and Mining Sciences, 38:297-310, 2001.

[19] C. Arson and B. Gatmiri. On damage modelling in unsaturated clay rocks. Physics and Chemistry of the Earth-Parts A/B/C, 33:S407-S415, 2008.

[20] C. Arson and J.-M. Pereira. Influence of damage on pore size distribution and permeability of rocks. International Journal for Numerical and Analytical Methods in Geomechanics, 37(8):810-831, 2013.

[21] S. C. Yuan and J. P. Harrison. Development of a hydro-mechanical local degradation approach and its application to modelling fluid flow during progressive fracturing of heterogeneous rocks. International Journal of Rock Mechanics and Mining Sciences, 42(7):961-984, 2005.

[22] J.-F. Shao, H. Zhou, and K. T. Chau. Coupling between anisotropic damage and permeability variation in brittle rocks. International Journal for Numerical and Analytical Methods in Geomechanics, 29(12):1231-1247, 2005.

[23] K. Maleki and A. Pouya. Numerical simulation of damage-permeability relationship in brittle geomaterials. Computers and Geotechnics, 37(5):619-628, 2010.

[24] A. Pandolfi, S. Conti, and M. Ortiz. A recursive-faulting model of distributed damage in confined brittle materials. Journal of Mechanics and Physics of Solids, 54:1972-2003, 2006.

[25] D. T. Snow. A parallel plate model of fractured permeable media. PhD thesis, University of California at Berkeley, California, 1965.

[26] D. T. Snow. Anisotropic permeability of fractured media. Water Resources Research, 5(6):1263-1279, 1969.

[27] R. C. Parsons. Permeability of idealized fractured rocks. Journal of the Society of Petroleum Engineers, 6:126-136, 1966.

[28] M. Ortiz and A. Pandolfi. A class of cohesive elements for the simulation of three-dimensional crack propagation. International Journal for Numerical Methods in Engineering, 44:1267-1282, 1999.

[29] A. Pandolfi, C. Kane, J. E. Marsden, and M. Ortiz. Time-discretized variational formulation of non-smooth frictional contact. International Journal for Numerical Methods in Engineering, 53(4):1801-1829, 2002.

[30] M. Ortiz and L. Stainier. The variational formulation of viscoplastic constitutive updates. Computer Methods in Applied Mechanics and Engineering, 171:419-444, 1999.

[31] J. Kozeny. Ueber kapillare leitung des wassers im boden. Sitzungsber Akad. Wiss., 136(2a):36, 1927.

[32] P. C. Carman. Fluid flow through granular beds. Transactions, Institution of Chemical Engineers, $15: 17,1937$.

[33] P. C. Carman. Flow of gases through porous media. London, 1956. 
[34] A. B. Göktepe and A. Sezer. Effect of particle shape on density and permeability of sands. Proceedings of the Institution of Civil Engineers: Geotechnical Engineering, 163:307-320, 2010.

[35] A. Sanzeni, F. Colleselli, and D. Grazioli. Specific surface and hydraulic conductivity of fine-grained soils. Journal of Geotechnical and Geoenvironmental Engineering, 139:1828-1832, 2013.

[36] M. G. Schaap and I. Lebron. Using microscope observations of thin sections to estimate soil permeability with the Kozeny-Carman equation. Journal of Hydrology, 251:186-201, 2001.

[37] Lasowska A. Ciesslicki, K. and A. Z. Smolarski. The influence of channel tortuousity on hydraulic resistance. Proceedings of the Institution of Civil Engineers: Geotechnical Engineering, 48:161-173, 2000.

[38] K. P. Saripalli, R. J. Serne, P. D. Meyer, and B. P. McGrail. Prediction of diffusion coefficients in porous media using tortuosity factors based on interfacial areas. Ground Water, 40:346-352, 2002.

[39] G. E. Archie. The electrical resistivity $\log$ as an aid in determining some reservoir characteristics. In SPE Reprint Series, pages 9-16. 2003.

[40] A. J. Katz and A. H. Thompson. Prediction of rock electrical conductivity from mercury injection measurements. Journal of Geophysical Research, 92:599-607, 1987.

[41] L. M. Schwartz, P. N. Sen, and D. L. Johnson. Novel geometrical effects in electrolytic conduction in porous media. Physica A. Statistical Mechanics and its Applications, 157:493-496, 1989.

[42] A. Revil and L. M. Cathles. Permeability of shaly sand. Physica A: Statistical Mechanics and its Applications, 35:651-662, 1999.

[43] F. Civan. Scale effect on porosity and permeability: Kinetics, model, and correlation. AIChE Journal, 47:271-287, 2001.

[44] F. Civan. Relating permeability to pore connectivity using a power-law flow unit equation. Petrophysics, 43:457-476, 2002.

[45] R. M. Cosentini, G. Della Vecchia, S. Foti, and G. Musso. Estimation of the hydraulic parameters of unsaturated samples by electrical resistivity tomography. Géotechnique, 62(7):583-594, 2012.

[46] O. Schulze, T. Popp, and H. Kern. Development of damage and permeability in deforming rock salt. Engineering Geology, 61(2):163-180, 2001

[47] S. Irmay. Flow of liquid through cracked media. Bulletin of the Research Council of Istrael, 1(5A):84, 1937.

[48] A. Peirce and E. Detournay. An implicit level set method for modeling hydraulically driven fractures. Computer Methods in Applied Mechanics and Engineering, 197(33):2858-2885, 2008.

[49] W. Chen and G. Ravichandran. Dynamic compressive behavior of ceramics under lateral confinement. Journal de Physique IV, 4:177-182, 1994

[50] W. Chen and G. Ravichandran. Static and dynamic compressive behavior of aluminum nitride under moderate confinement. Journal of the American Society of Ceramics, 79(3):579-584, 1996.

[51] W. Chen and G. Ravichandran. An experimental technique for imposing dynamic multiaxial compression with mechanical confinement. Experimental Mechanics, 36(2):155-158, 1996.

[52] W. Chen and G. Ravichandran. Failure mode transition in ceramics under dynamic multiaxial compression. International Journal of Fracture, 101:141-159, 2000.

[53] L. K. Ma, J. Wang, X. G. Zhao, and L. G. Tham. Experimental study on permeability of Beishan granite. In Rock mechanics: achievements and ambitions. Taylor \& Francis Group, London, 2012.

[54] W. Zhu and T.-F. Wong. The transition from brittle faulting to cataclastic flow: Permeability evolution. Journal of Geophysical Research, 102:3027-3041, 1997.

[55] J. Heiland. Laboratory testing of coupled hydro-mechanical processesduring rock deformation. Hydrogeology Journal, 11:122$141,2003$.

[56] N. R. Warpinski, R. C. Kramm, J. R. Heinze, and C. K. Waltman. Comparison of single- and dual-array microseismic mapping techniques in the barnett shale. In SPE Annual Technical Conference and Exhibition, volume SPE 95568, Dallas, Texas, October 2005. Society of Petroleum Engineers.

[57] R. Wu, O. Kresse, X. Weng, C. E. Cohen, and H. Gu. Modeling of interaction of hydraulic fractures in complex fracture networks. In SPE Hydraulic Fracturing Technology Conference, volume SPE-152052-MS, The Woodlands, Texas, February 2012. Society of Petroleum Engineers. 